\title{
Vergabe der Aufträge von Kunsttransporten im Leihverkehr
}

Bundeskartellamt, 1. Vergabekammer des Bundes, Beschluss vom 3. September 2009 - VK 1-155/09

\section{Zur Auslegung einzelner Tatbestandsmerkmale bei der Vergabe des Auftrages von Kunsttransporten nach VOL/A im Leihverkehr zwischen privatem Leihgeber und öffentlichem Leihnehmer. (Leitsatz der Redaktion)}

\section{Beschluss}

- In dem Nachprüfungsverfahren der

A.

- Antragstellerin -

(...)

gegen

B.

- Antragsgegnerin -

(...)

wegen der Vergabe des Auftrages von Kunsttransporten für die (...) (Vergabe Nr. [...]) hat die 1. Vergabekammer des Bundes durch den Vorsitzenden Leitender Regierungsdirektor Behrens, die hauptamtliche Beisitzerin Regierungsdirektorin Dr. Dittmann und den ehrenamtlichen Beisitzer Hagen auf die mündliche Verhandlung vom 25. August 2009 am 3. September 2009 beschlossen:

1. Es wird festgestellt, dass die von der Antragsgegnerin abgeschlossenen Verträge über Transportleistungen für die Exponate aus (...) (Leihgeber 1, 2, 3, 40, 41, 42, außer dem Exponat mit der Reg.-Nr. x0851 des Leihgebers 40) unwirksam sind.

2. Im Übrigen wird der Nachprüfungsantrag zurückgewiesen.

3. Die Kosten des Nachprüfungsverfahrens (Gebühren und Auslagen) trägt die Antragstellerin zu vier Fünfteln und die Antragsgegnerin zu einem Fünftel. Die Antragsgegnerin hat ein Fünftel der zur zweckentsprechenden Rechtsverfolgung notwendigen Aufwendungen der Antragstellerin zu tragen. Die Antragstellerin hat vier Fünftel der zur zweckentsprechenden Rechtsverteidigung notwendigen Aufwendungen der Antragsgegnerin zu tragen. Im Übrigen tragen die Verfahrensbeteiligten ihre Aufwendungen selbst.

4. Die Zuziehung eines Rechtsanwalts durch die Antragstellerin und die Antragsgegnerin war notwendig.

\section{Gründe:}

I.

1. Die Antragsgegnerin (Ag) schrieb die Vergabe des Auftrages von Kunsttransporten für die (...) (Vergabe Nr. [...]) mit Bekanntmachung vom (...) im Offenen Verfahren europaweit aus. Diese (...) hat am (...) 2009 in (...) begonnen und wird anschließend ab Mai 2010 in (...) und ab Oktober 2010 in (...) gezeigt (Ziff. 4.1.1 der Leistungsbeschreibung).

Der ausgeschriebene Auftragsgegenstand umfasst die Demontage, das Verpacken, den Transport, das Auspacken und die Installation der auszustellenden Exponate (Gemälde, Grafiken, Skulpturen sowie weitere Sammlungsgegenstände) von ursprünglich 42 Leihgebern zur Ag nach (...) sowie anschließend nach (...) und (...). Die Ausstellungsgegenstände sollten ursprünglich aus (...) (Leihgeber Nr. 1 - 3), (...) (Leihgeber Nr. 4, 5), (...) (Leihgeber Nr. 6- 18), (...) (Leihgeber Nr. 19), (...) (Leihgeber Nr. 20), (...) (..., Leihgeber Nr. 21), (...) (Leihgeber Nr. 22 - 38) und (...) (Leihgeber Nr. 39 - 42) stammen. Die Anlieferung in (...) sollte vom (...) bis (...) 2009 erfolgen (Ziff. Il.1.5 der Bekanntmachung, Ziff. 3.2, 3.8 der Leistungsbeschreibung). Die Bindefrist sollte am 24. Juli 2009 enden (Ziff. IV.3.7 der Bekanntmachung).

In der Bekanntmachung im Amtsblatt der EU sowie den Verdingungsunterlagen hatte die Ag die Vorlage einiger Unterlagen und Belege mit dem Angebot verlangt, u.a. in Ziff. III.2.3 der Bekanntmachung eine Zertifizierung nach DIN ISO 9000:2000ff für Kunsttransporte.

Nachdem sich der größte Leingeber aus (...) auf eine bestimmte Spedition festgelegt hatte, entschied die Ag am 10. Juni 2009, den Auftrag für zwei weitere Leihgeber aus (...) an denselben Spediteur zu vergeben, da kein wirtschaftlicheres Angebot zu erwarten sei. Nachdem ein Leihgeber aus (...) ebenfalls einen bestimmten Spediteur vorgegeben hatte, entschied die Ag am selben Tag, diesen Spediteur auch mit dem Antransport der Exponate der beiden weiteren Leihgeber aus (...) zu beauftragen, da ein günstigeres Angebot unwahrscheinlich sei. Außerdem entschied die Ag am 10. Juni 2009, „aufgrund der unerwartet langwierigen Beantragungsfristen" für erforderliche Ausfuhrgenehmigungen einen bestimmten (...) Spediteur bereits mit der Vorbereitung dieser Dokumente zu beauftragen (s. Vermerk Ag vom 16. Juni 2009).

Am 24. Juni 2009 fand ein Bietergespräch statt, in dem die Ag den Bietern (darunter die Antragstellerin [Ast] und die Beigeladene $[\mathrm{Bg}])$ u.a. mitteilte, dass Leihgaben aus (...) und (...) 
nicht mehr Gegenstand des ausgeschriebenen Auftrags seien (s. Protokoll des Bietergesprächs vom 24. Juni 2009). Außerdem wurde den Bietern eine überarbeitete Exponateliste übergeben - die ASt erhielt diese per E-Mail am 30. Juni 2009 -, auf der u.a. die Leihgaben aus den (...), (...) und (...) als „Storno Auftragsteil" (..., z.T. ... [Leihgeber 1, 2, 3, 40, 41, 42, außer dem Exponat ... des Leihgebers 40]) bzw. "Objekte abgesagt" (... [Leihgeber 29]), z.T. ... (Leihgeber 39 sowie das Exponat ... des Leihgebers 40) gekennzeichnet waren.

U.a. die ASt, die Bg sowie weitere Bieter gaben fristgerecht bis zum 17. Juli 2009, 10 Uhr, Angebote ab. In dem von der Ag vorbereiteten Formblatt "Kostenzusammenstellung" hatten weder die ASt noch die Bg mehr Preise zu den Leihgebern aus (...), (...) und (...) angegeben. Keines der Angebote enthielt sämtliche der vorzulegenden Eignungserklärungen und -nachweise, das Angebot der ASt enthielt u.a. kein DIN ISO-Zertifikat für Kunsttransporte. Außerdem hatten mehrere Bieter ihrem Angebot eigene Allgemeine Geschäftsbedingungen beigefügt.

Am 21. Juli 2009 teilte die Ag der ASt mit, dass sie ihr Angebot nicht berücksichtigen könne, da es das geforderte DIN ISO-Zertifikat für Kunsttransporte nicht enthalte; der Zuschlag solle der Bg erteilt werden. Mit Rügen vom 23. und 30. Juli 2009 wandte sich die ASt gegen den Ausschluss ihres Angebots wegen des fehlenden Zertifikats und beanstandete hierüberhinaus u.a., dass die Ag den ausgeschriebenen Leistungsumfang nachträglich "mindestens um die Kunsttransporte aus (...) und (...) in erheblichem Umfang reduziert" und nach (...) und (...) eine "Direktbeauftragung" vorgenommen habe. Nachdem die Ag diesen Rügen nicht abgeholfen hatte, stellte die ASt am 30. Juli 2009 einen Nachprüfungsantrag. Dieser Antrag wurde der Ag am 31. Juli 2009 zugestellt.

Mit Schreiben vom 10. August 2009 teilte die Ag den Bietern mit, dass sie die Ausschreibung gemäß § $26 \mathrm{Nr}$. 1 lit. a) VOL/A aufhebe, es sei kein Angebot eingegangen, das den Ausschreibungsbedingungen entspreche. Aufgrund der Eilbedürftigkeit solle der Antransport der Leihgaben nach (...) kurzfristig einzeln vergeben und der Rest der Ausstellungstournee erneut ausgeschrieben werden. Die Aufhebung wurde am 10. August 2009 im Amtsblatt der EU veröffentlicht.

Hinsichtlich des Antransports von Leihgaben nach (...) legt die Ag im Nachprüfungsverfahren folgende Unterlagen vor:

- Ein Schreiben vom 11. August 2009, mit dem der Auftrag für den Antransport aus (...) erteilt wird;

- ein Schreiben vom 11. August 2009 bzgl. der Auftragserteilung des Antransportes aus zwei Museen in (...);

- ein Schreiben vom 12. August 2009 über den Antransport aller Ausstellungsgegenstände von (...) Leihgebern („we would like to confirm the order [...]");
- sowie ein Schreiben vom 12. August 2009 über den Auftrag für den Antransport der (...) und der (...) Leihgaben zur Ag.

Auftragnehmer waren insbesondere solche Unternehmen, die von mehreren Bietern als Nachunternehmer benannt worden waren.

Am 12. August 2009 rügte die ASt, dass der Antransport nach (...) kurzfristig einzeln vergeben wurde, die Leistungen seien mindestens teilweise bereits erbracht worden.

2. Im Nachprüfungsverfahren haben die Verfahrensbeteiligten Folgendes vorgetragen:

a) Die ASt stellt unstreitig, dass kein Angebot den Ausschreibungsbedingungen entsprach, weist jedoch darauf hin, dass die Ag zur geforderten Zertifizierung nach DIN ISO zumindest einen gleichwertigen Qualitätsnachweis hätte zulassen müssen.

Die Herausnahme der Exponate aus (...), (...) und (...) aus dem ursprünglich ausgeschriebenen Leistungskatalog sowie die anschließende Direktvergabe dieser Leistungen hält die ASt für vergaberechtswidrig. Zur Rechtzeitigkeit ihrer entsprechenden Rüge trägt die ASt vor, dass sie aufgrund des Bietergesprächs am 24. Juni 2009 davon ausgegangen sei, dass nur das Besorgen der Exportlizenzen beim Auftrag "(...)", jedoch nicht der Transport der Exponate selbst, sowie die Exponate aus (...) nicht mehr Gegenstand der Ausschreibung seien. Die ASt führt aus, dass sie bereits am Tag des Bietergesprächs ihre Agenten in den (...) und (...) informiert habe, dass die Leihgaben aus diesen Ländern aus der Ausschreibung herausgenommen worden seien. Am folgenden Tag hätten diese Unternehmen der ASt mitgeteilt, dass sie von der Ag bereits in der vorhergehenden Woche um direkte Angebote gebeten worden seien. Erst in der Woche, in der die ASt ihren Nachprüfungsantrag gestellt habe, habe sie erfahren, dass die Ag den herausgenommen Kunsttransporteanteil aus (...) und den (...) bereits direkt vergeben hatte; dass dies vergaberechtswidrig sei, habe die ASt erst aufgrund der unmittelbar vor Stellung des Nachprüfungsantrags erfolgten anwaltlichen Beratung gewusst.

Hinsichtlich des Antransports der übrigen Leihgaben nach (...) meint die ASt, dass die Ag bereits vor der Aufhebung der Ausschreibung Aufträge für sämtliche dieser Leistungen erteilt habe. Es sei aus logistischen und organisatorischen Gründen so gut wie gar nicht möglich, Leistungen ab der vermeintlichen Auftragserteilung vom 11. bzw. 12. August 2009 so zu erbringen, dass die Ausstellung in (...) ungefährdet in vollem Umfang eröffnet werden könne. Die Aufhebung der Ausschreibung sei daher insoweit rechtswidrig, zudem seien die bereits erteilten Aufträge vergaberechtswidrig und deshalb unwirksam. Die Dokumentation der Ag hierzu sei offensichtlich unzureichend; einer solchen Auftragserteilung müssten vorbereitende E-Mails oder anderer Schriftverkehr vorangegangen sein, der nicht aktenkundig sei. Die Dokumentation dürfe auch nicht erst nach Abschluss des 
Vergabeverfahrens und Zuschlagserteilung angefertigt werden, Dokumentationsmängel dürften auch nicht durch Sachvortrag im Nachprüfungsverfahren behoben werden.

\section{Im Einzelnen führt die ASt insoweit Folgendes aus:}

- Hinsichtlich der Antransporte aus (...) habe die ASt bereits am 11. August 2009, also einen Tag nach der schriftlichen Mitteilung über die Ausschreibungsaufhebung, festgestellt, dass ein erster Truck mit Leihgaben bereits am selben Nachmittag in (...) eintreffen sollte. Alle übrigen Leihgaben aus (...) seien für den Transport am 18. August 2009 bzw. 24. August 2009 geplant gewesen. Die Aufträge für diese Antransporte hätten deshalb zwingend schon vor der Aufhebung der Ausschreibung erteilt worden sein müssen. Dies werde durch das Schreiben der Ag an den Auftragnehmer vom 12. August 2009 bestätigt, bei dem es sich offensichtlich lediglich um die Bestätigung einer bereits vorher erteilten "Order" zum Transport handele (,[...] to confirm the order [...]"). Die ASt legt eine E-Mail aus (...) vom Vormittag des 11. August 2009 vor, in der berichtet wird, dass Leistungen für Leihgaben aus (...) nach (...) am 11. August bereits ausgeführt gewesen seien. Dieser E-Mail lasse sich ebenfalls entnehmen, dass alle Transportkisten bereits gebaut gewesen waren und die verlangten Packmaterialien bereits geliefert waren. Der entsprechende Auftrag müsse auch aus diesem Grund bereits früher erfolgt sein, denn es sei nicht anzunehmen, dass das Transportunternehmen in einer Größenordnung von (...) ins Blaue hinein plane und Kisten baue.

- Beim Auftrag für (...) gehe es immerhin um knapp 90 Leihgaben, für die mindestens 11 Klimakisten benötigt werden würden, die erst gebaut bzw. besorgt werden müssten, um sodann diese Leihgaben zu verpacken etc. Am 12. August seien diese Kisten jedoch bereits an die betreffenden (...) Museen angeliefert worden, die Kisten wurden am 13. und 14. August bepackt und am 17. August 2009 habe der Transport von (...) nach (...) stattgefunden. Die ASt legt dar, dass dies ihrer Auffassung nach schlechterdings nicht „über Nacht" erfolgt sein könne.

- Einen Auftrag für einen Antransport aus (...) nach (...) habe die Ag schließlich überhaupt nicht vorgelegt, obwohl auch von dort An- und Abtransporte vorgesehen seien.

Sofern Antransportleistungen nach (...) nach Aufhebung der Ausschreibung überhaupt noch zu beauftragen gewesen seien - was nicht der Fall sei -, hätten diese auch nicht wegen Eilbedürftigkeit sofort vergeben werden dürfen. Vielmehr hätte die Ag diese Antransportleistungen aus verschiedenen Ländern europaweit ausschreiben müssen. So sei der erforderliche Schwellenwert überschritten, da allein schon der Wert dieser Transportleistungen nach - bestrittener - Mitteilung der Ag insgesamt ca. $160.000 €$ betragen hätte, hinzu kämen die weiteren Transportleistungen zu den anderen Ausstellungsorten und der
Bereich (...). Die ASt meint, dass die losweise Vergabe der Antransportleistungen gemäß §§ 2, 3 VgV für die Berechnung des Schwellenwertes nicht isoliert betrachtet werden dürften. Vielmehr handele es sich um den einheitlichen Ausstellungsgegenstand (...), der an mehreren Ausstellungsorten stattfinden solle. Aus diesem Grund habe die Ag die entsprechenden Leistungen ursprünglich auch einheitlich europaweit ausgeschrieben. Außerdem lägen die von der Ag behaupteten Voraussetzungen für ein freihändiges Verfahren bzw. ein Verhandlungsverfahren nicht vor. Die „Eilbedürftigkeit" iSd § 3a Nr. 2 lit. d) VOL/A sei von der Ag selbst verschuldet gewesen, da diese nicht bereits bei der Angebotswertung am 20. Juli, sondern erst am 10. August 2009 bemerkt habe, dass alle Angebote zwingend ausgeschlossen werden mussten. Im Zeitpunkt der Wertung der Angebote wäre ein beschleunigtes Verfahren mit Fristen nach § 18a VOL/A ohne Weiteres noch möglich gewesen. Für die Fertigstellung der zu erbringenden Leistungen sei auch nicht wie ursprünglich ausgeschrieben auf den 8. August 2009 abzustellen, denn wie die von der Ag vorgelegten Auftragsschreiben zeigten, reiche anscheinend auch eine Beauftragung am 12. August aus, um die Ausstellung rechtzeitig vorbereiten zu können. Auch insoweit fehle es an jeglicher Dokumentation in der Vergabeakte, vielmehr dokumentiere die Ag, über die einschlägigen Tatbestandsvoraussetzungen des § 3a Nr. 2 VOL/A gar nicht nachgedacht zu haben. Die ASt meint außerdem, dass die Ag auch bei einer freihändigen Vergabe einen Wettbewerb hätte veranstalten und bei zumindest drei Unternehmen um Angebote hätte anfragen müssen. Insoweit sei nicht nachzuvollziehen, dass die Ag nicht die bisherigen Bieter in diese Neuausschreibung mit einbezogen habe, diese hätten sich mehr sogar als die tatsächlich von der Ag beauftragten Nachunternehmer der bisherigen Bieter bereits auf die Durchführung des Auftrags vorbereitet. Die ASt bestreitet, dass alle Bieter jeweils nur dieselben Nachunternehmer angegeben hätten, so dass die Ag direkt gerade auf diese hätte zugehen können.

Die ASt ist der Auffassung, sie habe ein berechtigtes Interesse, die Rechtswidrigkeit der Vorgehensweise der Ag feststellen zu lassen, da sie ihr Interesse an etwaigen, noch offenen Antransportleistungen durch die Teilnahme am vorangegangenen Vergabeverfahren bereits bekundet habe und auch ein berechtigtes Interesse an einer ordnungsgemäßen Teilaufhebung des bisherigen Vergabeverfahrens und an einer ordnungsgemäßen Neuausschreibung habe.

Abschließend trägt die ASt vor, dass die Ag die Kosten des Nachprüfungsverfahrens zu übernehmen habe, wie immer der Fall auch gelagert sei. Die Ag habe nach jeder Betrachtungsweise eine unzulässige Wartefrist für die Auftragserteilung an die Bg vorgesehen, indem sie dieser ohne Einhaltung der in § 101a GWB vorgeschriebenen Frist bereits am 24. Juli 2009 den Zuschlag habe erteilen wollen, so dass die Ag allein damit schon das Nachprüfungsverfahren provoziert habe. 


\section{Die ASt beantragt zuletzt:}

1. Es wird festgestellt, dass die Aufhebung des Vergabeverfahrens Nr. (... ) (...) der Ag insoweit vergaberechtswidrig gewesen ist bzw. ist, wie Aufträge, insbesondere zum Antransport nach (...), bereits an die Bg oder auch an Dritte erteilt worden sind, und durchgeführt worden sind bzw. aufgrund eines Auftrages sich gegenwärtig bereits in der Abwicklung befinden.

2. Es wird festgestellt, dass bereits erteilte Aufträge an die Bg oder an Dritte, insbesondere zum Antransport, vergaberechtswidrig und von Anfang an unwirksam sind.

3. Es wird festgestellt, dass eine Neuausschreibung bzw. Vergabe der Antransportleistungen der Leihgaben nach (...) (zur Ausstellungseröffnung am [... 2009) nicht mit der Begründung der Eilbedürftigkeit vergaberechtsgemäß erfolgen konnte bzw. kann.

4. Es wird festgestellt, dass das Vergabeverfahren Nr. (...) nicht ordnungsgemäß dokumentiert und daher insoweit vergaberechtswidrig ist.

5. Die Ag trägt die Kosten des Nachprüfungsverfahrens und die der ASt entstandenen Rechtsanwaltskosten.

b) Die Ag beantragt,

1. den Nachprüfungsantrag zurückzuweisen;

2. festzustellen, dass die Hinzuziehung eines Verfahrensbevollmächtigten durch die Ag notwendig war.

Die Ag meint, die Nachprüfungsanträge der ASt seien bereits unzulässig, weil die ASt keine Chance auf eine Erteilung des Auftrags iSd § 107 Abs. 2 GWB habe. Denn das von der ASt vorgelegte Angebot sei aus mehreren Gründen von der Wertung ausgeschlossen.

Jedenfalls seien die Nachprüfungsanträge offensichtlich unbegründet, weil es im Zusammenhang mit der Aufhebung keine Verfahrensfehler gegeben habe. Die Ag habe erst aufgrund der anwaltlichen Beratung aus Anlass des Nachprüfungsverfahrens festgestellt, dass keines der Angebote alle formellen Vorgaben erfüllt.

Die Ag vertritt die Auffassung, dass die schon lange vor Einleitung dieses Nachprüfungsverfahrens und vor der Aufhebungsentscheidung der Ag gesondert beauftragten Anlieferungen aus (...), (...) und (...) für dieses Verfahren vollständig unerheblich seien. Der Leistungsumfang habe aus Gründen, die von der Ag nicht zu vertreten seien, reduziert werden müssen. Da die jeweiligen Leihgeber den Bau der erforderlichen Transportkisten zum Teil bereits veranlasst und bestimmte Spediteure beauftragt gehabt hätten, sei es für die Ag faktisch unmöglich gewesen, diese Leistungen so wie ursprünglich ausgeschrieben zu vergeben. Hierüber seien die Bieter in dem Bietergespräch am 24. Juni 2009 unter Übergabe einer entsprechend korrigierten Exponateliste informiert worden. Die ASt habe gegen diese Vorgehensweise keine Einwendungen erhoben, geschweige denn unverzüglich iSd § 107 Abs. 3 Nr. 1 GWB gerügt. Die Änderungen des ausgeschriebenen Leistungsumfangs hätten alle Bieter, auch die ASt, ihren Angeboten zugrundegelegt.

Die Auftragswerte für die einzeln vergebenen Antransporte nach (...) lägen auch zusammengerechnet weit unterhalb des EU-Schwellenwertes. Der Leistungsumfang habe sich bereits vor Ablauf der Angebotsfrist - ohne dass die ASt dies im Vergabeverfahren gerügt habe - durch von der Ag nicht zu vertretende Entscheidungen verschiedener Leihgeber, die eine Überlassung ihrer Exponate kurzfristig von dem Transport durch ein selbst ausgewähltes Unternehmen abhängig gemacht hätten, immer wieder verändert. Spätestens bei der Aufhebung der Ausschreibung am 10. August 2009 habe es daher zwar weiterhin kunsthistorisch, aber nicht mehr länger vergaberechtlich einen einheitlichen Ausstellungsgegenstand (...) gegeben. Aufgrund der besonderen Dringlichkeit der Leistung (die Ausstellung werde derzeit aufgebaut und am [...] eröffnet), sei gemäß § 3 Nr. 4 lit. f) VOL/A die Freihändige Vergabe gerechtfertigt gewesen. Wegen der Eilbedürftigkeit habe die Ag nur solche Unternehmen berücksichtigt, die von verschiedenen Bietern in dem aufgehobenen Verfahren schon als Subunternehmer vorgesehen gewesen waren. Nur diese Unternehmen hätten sich bereits im Rahmen der ursprünglichen Angebotsabgabe in ausreichendem Umfang mit dem Gegenstand des Auftrags befasst, um diesen nunmehr innerhalb kürzester Zeit selbst ausführen zu können. Außerdem sei es aus Kostengesichtspunkten sinnvoll gewesen, nur Unternehmen "vor Ort", also aus dem Land der jeweiligen Leihgeber, zu beauftragen und nicht noch dritte Unternehmen zum Verfahren hinzuzuziehen.

Selbst wenn die kurzfristig erforderlichen Beauftragung der Anlieferung nach (...) als EU-weites Verfahren iSd 2. Abschnitts der VOL/A zu beurteilen gewesen wäre, sei ein Verhandlungsverfahren ohne vorherige Bekanntmachung nach § 3a Nr. 2 lit. d) VOL/A zulässig, denn vorliegend seien die Besonderheiten des Kunsthandels zu berücksichtigen. Bei Ausstellungen mit fremden Leihgaben sei es üblich, dass sich der Umfang der Ausstellungsobjekte bis zum letzten Tag ändern könne. Der Leihgeber selbst dürfe entscheiden, was er wie zur Verfügung stelle.

Die Ag trägt vor, dass nach ihrer Kenntnis Leistungen, die Gegenstand der verfahrensgegenständlichen Ausschreibung waren, nicht schon vor der Aufhebung vom August 2009 erbracht worden seien. Etwaige Vorarbeiten der jeweiligen Leihgeber seien zumindest nicht im Auftrag der Ag erfolgt. Die Ag selbst habe sich vor dem 10. August 2009 nicht um einzelne Transportaufträge gekümmert, weil sie davon ausgegangen sei, dass ihr Vergabeverfahren ordnungsgemäß durchgeführt worden sei. 
Zur Anlieferung von Leihgaben aus (...) führt die Ag aus, dass über einen bestimmten Spediteur bereits im Juni 2009 die Vorbereitung der Ausfuhrgenehmigungen eingeleitet worden sei, da besondere Ausfuhrbestimmungen einzuhalten gewesen seien. Dies habe sich später aber als in diesem Umfang gar nicht erforderlich erwiesen. Das betreffende Transportunternehmen habe ziemlich sicher davon ausgehen können, in jedem Fall mittelbar an der Leistungserbringung beteiligt zu werden, da es offenbar von allen Bietern als Nachunternehmer angefragt worden sei (was für die ASt mangels entsprechender Angaben in deren Angebot nur vermutet werden könne). Nachdem die Daten zur Anlieferung der Exponate seit langem feststanden, habe dieser Spediteur in Zusammenarbeit mit den Leihgebern offenbar beträchtliche Vorarbeiten geleistet, um - auf wessen Rechnung letztlich auch immer - seine Transportleistung termingerecht erbringen zu können. In der mündlichen Verhandlung trägt die Ag vor, dass bereits am 11. August 2009 ein Lastwagen in (...) eingetroffen sei, der ausschließlich Leihgaben eines bestimmten (...) Museums antransportiert habe und nicht von der $\mathrm{Ag}$, sondern direkt von diesem Museum beauftragt worden sei. Dies sei auch bei anderen (...) Museen so geschehen.

Auch hinsichtlich des Antransports der Exponate aus (...), der (...) und (...) sei der ursprünglich von verschiedenen Bietern vorgesehene Nachunternehmer beauftragt worden, weil nur dieses Unternehmen aufgrund seines Vorwissens aus der ursprünglichen Angebotserstellung und aufgrund von Vorarbeiten, die von der Ag nicht vor dem 11. August 2009 beauftragt worden waren, die erforderliche kurzfristige Leistungserbringung gewährleisten konnte.

c) Durch Beschluss vom 7. August 2009 wurde die Bg zum Verfahren hinzugezogen. Diese hat keine schriftliche Stellungnahme abgegeben und beantragt in der mündlichen Verhandlung über ihre Verfahrensbevollmächtigten,

die Anträge der ASt abzuweisen und ihr die Kosten der Bg aufzuerlegen.

Der ASt wurde unter Beachtung von Geschäftsgeheimnissen Akteneinsicht gewährt. In der mündlichen Verhandlung am 25. August 2009 hatten die Beteiligten Gelegenheit, ihre Standpunkte darzulegen. Auf die ausgetauschten Schriftsätze, die Verfahrensakte der Vergabekammer sowie auf die Vergabeakten, soweit sie der Vergabekammer vorgelegen haben, wird ergänzend Bezug genommen. Der nicht nachgelassene Schriftsatz der ASt vom 25. August 2009 nach der mündlichen Verhandlung erfolgte im Hinblick auf die den Beteiligten obliegende Verfahrensförderungspflicht, § 113 Abs. 2 GWB, verspätet und bleibt bei der Entscheidungsfindung unbeachtet (vgl. OLG Düsseldorf, Beschlüsse vom 19. November 2003, VII-Verg 22/03; und vom 28. Juni 2006, VII-Verg 18/06).

\section{II.}

Der Beschluss ergeht auf der Grundlage des GWB in der Fassung der Bekanntmachung vom 15. Juli 2005 (BGBI. I S. 2114), zuletzt geändert durch das Gesetz zur Modernisierung des Vergaberechts vom 20. April 2009 (BGBI. I S. 790), da das dem Nachprüfungsverfahren zugrunde liegende Vergabeverfahren nach dem 24. April 2009 begonnen hat ( $\$ 131$ Abs. 8 GWB).

Der Nachprüfungsantrag ist z.T. bereits unzulässig, im Übrigen überwiegend unbegründet. Lediglich soweit es um die Vergabe der Transporte von Leihgaben aus den (...) und (...) geht (Leihgeber 1, 2, 3, 40, 41, 42, außer dem Exponat mit der Reg.-Nr. x0851 des Leihgebers 40), war die Vorgehensweise der Ag rechtswidrig, die von der Ag insoweit abgeschlossenen Verträge sind gemäß § 101 Abs. 1 Nr. 2 GWB unwirksam.

\section{Der Nachprüfungsantrag ist nur teilweise zulässig.}

a) Das Nachprüfungsverfahren ist zumindest hinsichtlich der Transportleistungen aus (...) und (...) statthaft, da der einschlägige Schwellenwert, § $2 \mathrm{Nr}$. $3 \mathrm{VgV}$ unstreitig überschritten ist. Auch die Ag selbst hat nach der Teilaufhebung hinsichtlich der Leistungen u.a. bezüglich der (...) und (...) nichts dazu vorgetragen, dass der durch die Konzeption der Ausstellung gegebene Zusammenhang zwischen den ursprünglich auch als Gesamtauftrag ausgeschriebenen Leistungen nachträglich entfallen sei - die Ag meint lediglich, dass eine Gesamtausschreibung dieser Leistungen aus bestimmten Gründen nicht mehr wirtschaftlich gewesen wäre.

Hinsichtlich des Transports der Leihgaben nach (...) von den übrigen Leihgebern kann offen bleiben, ob die einzelnen ursprünglich gemeinsam ausgeschriebenen Transportleistungen isoliert betrachtet werden dürfen und damit ggf. unterhalb des o.g. Schwellenwertes liegen. Der Nachprüfungsantrag ist nämlich bzgl. der Leihgaben aus (...) jedenfalls aus anderen Gründen unzulässig (s.u. unter c) und bzgl. des Antransports nach (...) im Übrigen jedenfalls unbegründet (dazu unter 2.).

b) Gegen die Antragsbefugnis der ASt iSd § 107 Abs. 2 GWB bestehen keine Bedenken, auch wenn ihr Angebot aus formalen Gründen zwingend bereits deshalb gemäß $\S 25$ Nr. 2 Abs. 1 VOL/A von der Wertung auszuschließen ist, weil es nicht sämtliche von der Ag geforderten Eignungsnachweise und -erklärungen enthält (s. nur OLG Düsseldorf, Beschluss vom 28. Juni 2006, VII-Verg 18/06). Die ASt beanstandet nämlich nicht deshalb die Aufhebung der Ausschreibung am 10. August 2009, um das aufgehobene Vergabeverfahren unter Einbeziehung ihres Angebots fortzuführen, sondern weil sie meint, dass die Aufhebung rechtswidrig war, weil die ausgeschriebenen Leistungen zu diesem Zeitpunkt von der Ag bereits anderweitig vergeben worden waren. Die Richtigkeit dieses Vorbringens unterstellt, spielt der Ausschluss des Angebots der ASt in dem aufgehobenen Vergabeverfahren keine Rolle, denn die ASt macht geltend, sie sei von der Ag rechtswidrig nicht an dieser anderweitigen 
Vergabe beteiligt worden. Durch diese Nichtbeteiligung habe sie keine Chance gehabt, ein neues, nicht auszuschließendes Angebot einzureichen. Ein drohender Schaden ist damit hinreichend dargelegt (vgl. zu einer ähnlichen Sachverhaltskonstellation BGH, Beschluss vom 26. September 2006, X ZB 14/06).

c) Soweit es um die Herausnahme der Antransportleistungen der Exponate aus (...) (Leihgeber 20) und das Exponat (...) aus (...) (Leihgeber 40) aus dem ursprünglich ausgeschriebenen Leistungsumfang geht, ist der Nachprüfungsantrag jedoch mangels rechtzeitiger Rüge der ASt unzulässig. Aufgrund des Bietergesprächs am 24. Juni 2009 sowie spätestens aus der der ASt am 30. Juni 2009 zugegangenen aktualisierten Exponateliste war für die ASt erkennbar, dass die gemäß Ziff. 3.2 der Leistungsbeschreibung ursprünglich zu transportierenden Gegenstände aus (...) nicht mehr Gegenstand der Ausschreibung sein sollten (s. die überarbeitete Exponateliste zu den Leihgebern 20 und 40: „Objekte abgesagt“). Dementsprechend hat die ASt hierzu auch kein Angebot mehr abgegeben, indem sie die entsprechenden Teilleistungen in ihrem Angebot nicht bepreist hat. Diese Leistungen wurden von der Ag auch nicht anderweitig vergeben. Abgesehen davon, dass ein Bieter keinen Anspruch darauf hat, dass ein öffentlicher Auftraggeber bestimmte Leistungen überhaupt vergibt, selbst wenn er zuvor ein entsprechendes Vergabeverfahren eingeleitet hat (vgl. nur BGH, Beschluss vom 18. Februar 2003, X ZB 43/02), ist die ASt mit ihrem Vorbringen zur Rechtswidrigkeit der Reduzierung des Leistungsumfangs insoweit präkludiert, da diese (angeblichen) Vergaberechtsverstöße für sie bereits aus den Vergabeunterlagen erkennbar waren und von ihr nicht bis zum Ablauf der in der Bekanntmachung genannten Angebotsfrist am 17. Juli 2009 gerügt wurden, § 107 Abs. 3 Nr. 3 GWB.

Auch die Herausnahme der Transportleistungen aus (...) und (...) (Leihgeber 1, 2, 3, 40, 41, 42, außer dem Exponat mit der Reg.-Nr. x0851 des Leihgebers 40) war für die ASt bereits aus den Vergabeunterlagen erkennbar und wurde von ihr entgegen $\S 107$ Abs. 3 Nr. 3 GWB nicht bis zum Ablauf der Angebotsfrist gerügt. Diesbezüglich beanstandet die ASt jedoch nicht nur die nachträgliche Reduzierung des ausgeschriebenen Leistungsumfangs, die im Wege einer Teilaufhebung des bisherigen Vergabeverfahrens erfolgte. Vielmehr geht es der ASt hierüber hinaus auch darum, dass sie an der unstreitig durchgeführten Vergabe dieser Leistungen an von den Leihgebern vorbestimmte Auftragnehmer selbst nicht (mehr) beteiligt worden ist. Dies war für die ASt allein anhand der Vergabeunterlagen nicht erkennbar. Für die Rechtzeitigkeit ihrer Rüge am 30. Juli 2009 nach entsprechender anwaltlicher Beratung kommt es deshalb gemäß § 107 Abs. 3 Nr. 1 GWB auf die positive Kenntnis der ASt vom gerügten Vergaberechtsverstoß an. Zwar ist der Vortrag der ASt selbst hierzu widersprüchlich. So trägt sie kurz vor der mündlichen Verhandlung sowie in dieser selbst vor, dass sie zunächst noch davon ausgegangen sei, dass lediglich das Besorgen der Exportlizenzen beim Auftrag "(...)" schon anderweitig vergeben wurde, jedoch nicht der Transport der Exponate aus (...) selbst - dem stehen jedoch ihr Angebot selbst, ihre Rüge vom 30. Juli 2009 und ihr Vorbringen im Nachprüfungsantrag selbst entgegen, wonach sie überhaupt keine Leistungen (auch nicht für den Transport) für Leihgaben aus den (...) und (...) mehr angeboten und sie nach Gesprächen mit ihren Agenten in (...) und (...) die unmittelbare Vergabe an bestimmte Spediteure gerade als vergaberechtswidrig beanstandet hat. Unabhängig davon, welcher Einlassung der ASt man folgt, spricht jedoch nichts dafür, dass die ASt über diese Fakten hinaus auch die Rechtswidrigkeit der behaupteten Vergaberechtsverstöße bereits vor der anwaltlichen Beratung positiv erkannt und gleichwohl nicht unverzüglich gerügt hat (vgl. zu den Anforderungen an das Vorliegen einer "positiven Kenntnis" nur BGH, Beschluss vom 26. September 2006, aaO).

Was den Antransport der Kunstgegenstände von den weiteren Leihgebern angeht, bestehen hinsichtlich der Erfüllung der Rügeobliegenheiten der ASt keine Bedenken. Denn die Aufhebung der ursprünglichen Ausschreibung erfolgte erst am 10. August 2009, also während des laufenden Nachprüfungsverfahrens. Hinsichtlich ihrer hiermit zusammenhängenden Beanstandungen ist eine Rüge der ASt somit entbehrlich (vgl. BGH, Beschluss vom 26. September 2006, aaO).

\section{Der Nachprüfungsantrag ist überwiegend unbegründet.}

a) Die Ag hat die Transportleistungen aus (...) und (...) (Leihgeber 1, 2, 3, 40, 41, 42, außer dem Exponat mit der Reg.-Nr. x0851 des Leihgebers 40) nachträglich aus dem ursprünglich ausgeschriebenen Leistungsumfang (im Wege einer Teilaufhebung des ursprünglichen Vergabeverfahrens) herausgenommen und an Auftragnehmer vergeben, die die jeweiligen Leihgeber festgelegt hatten, bzw., soweit keine entsprechenden Vorgaben durch die Leihgeber erfolgt waren, auch die übrigen Leihgaben aus diesen Ländern an dieselben Spediteure vergeben, weil sie der Auffassung war, eine gesonderte Ausschreibung würde zu keinem wirtschaftlichen Ergebnis führen. Dies war vergaberechtswidrig:

Die Voraussetzungen für eine Vergabe im Verhandlungsverfahren ohne vorherige Bekanntmachung nach § 3a Nr. 2 lit. d) VOL/A (Dringlichkeit) liegen insoweit nicht vor, weil im Zeitpunkt der Vergabeentscheidung (10. Juni 2009) noch hinreichend Zeit für die Durchführung eines Vergabeverfahrens innerhalb der (ggf. verkürzten) Fristen des § 18a VOL/A gegeben war.

Die Voraussetzungen des für ein solches Verfahren allenfalls noch in Betracht kommenden $\S 3 a \mathrm{Nr} .2$ lit. c) VOL/A liegen insoweit ebenfalls nicht vor. Dieser Ausnahmetatbestand vom Grundsatz des Offenen Verfahrens ist eng auszulegen. Zum Einen stellt allein der Wunsch bzw. die Vorgabe eines Leihgebers, einen bestimmten Spediteur zu beauftragen und anderenfalls die Überlassung der Leihgaben zu verweigern, keine „technische oder künstlerische Besonderheit“ iSd § 3a Nr. 2 lit. c) VOL/A dar. Zwar sieht die Vergabekammer die praktischen Schwierigkeiten $\operatorname{der} \mathrm{Ag}$, die zumindest bei privaten Leihgebern oder bei solchen, die aus anderen Gründen nicht ans EU-Vergaberecht gebunden 
sind, keine Möglichkeit hat, deren Praxis bei der Erteilung von Transportaufträgen zu beeinflussen, und dennoch nicht auf deren Leihgaben verzichten möchte. Sollte jedoch allein das Verhalten eines nicht ans Vergaberecht gebundenen Dritten eine "Besonderheit" iSd § 3a Nr. 2 lit. c) VOL/A sein können, so dass Verträge unter den geringen Anforderungen des § $3 a$ Nr. 1 Abs. 1, Abs. 2 lit. c) VOL/A direkt vergeben werden dürften, würde dies dem vergaberechtlichen Wettbewerbsgrundsatz und dem grundsätzlichen Vorrang des Offenen Verfahrens im Sinne der gebotenen engen Auslegung der Ausnahmetatbestände des $\S$ 3a Nr. 2 VOL/A zuwiderlaufen. Diese Transportaufträge können auch nicht "aufgrund des Schutzes eines Ausschließlichkeitsrechts" iSd § 3a Nr. 2 lit. c) VOL/A nur von einem bestimmten Unternehmen ausgeführt und aus diesem Grund im Verhandlungsverfahren vergeben werden. Von diesem Tatbestand umfasst sind nämlich lediglich etwaige Ausschließlichkeitsrechte des Auftragnehmers selbst, nicht jedoch - wie hier - allenfalls das Ausschließlichkeitsrecht (Eigentum) des Leihgebers, der dem Auftragnehmer lediglich sein Eigentum zur Durchführung einer Transportleistung zur Verfügung stellt.

Die von der Ag dementsprechend abgeschlossenen Verträge sind somit gemäß § 101b Abs. 1 Nr. 2 GWB unwirksam.

b) Soweit es um den Antransport der Leihgaben nach (...) aus den übrigen Ländern geht, liegen jedoch keine Vergaberechtsverstöße seitens der Ag vor.

aa) Es ist nicht belegt, dass diese Aufträge bereits vor der Aufhebung der Ausschreibung am 10. August 2009 vergeben wurden. Die Ag hat Auftragsschreiben vorgelegt, ausweislich denen erst am 11. August 2009 Aufträge für den Transport der ausgeschriebenen Exponate aus (...) und (...) erteilt wurden bzw. am 12. August 2009 für den Transport von Exponaten aus (...), (...) und (...). Die ASt hat nichts vorgetragen, was diese Aktenlage widerlegt. So hat die Ag erklärt, dass der von der ASt angesprochene Lieferwagen aus (...) nicht aufgrund einer Beauftragung durch die Ag selbst, sondern aufgrund der Initiative des betreffenden (...) Leihgebers bereits am 11. August 2009 in (...) eingetroffen sei. Ebenso verhält es sich mit den von der ASt erwähnten Leihgaben aus (...), so dass die nach Auffassung der ASt ungewöhnlich schnelle Verpackung und Anlieferung dieser Leihgaben nicht zwingend belegt, dass die Ag selbst diese Leistungen bereits vor dem 10. August 2009 in Auftrag gegeben hätte. Dass das betreffende (...) Speditionsunternehmen auch ohne Auftrag der Ag rechtzeitig für eine solche Lieferung vorbereitet war, ist nach dem unstreitigen Sachvortrag der Verfahrensbeteiligten damit zu erklären, dass gerade dieser Spediteur auch schon im Juni 2009 mit der Vorbereitung bestimmter Ausfuhrgenehmigungen betraut worden war. Da es sich bei den meisten der jetzigen Auftragnehmer um solche Spediteure handelt, die von mehreren Bietern auch schon als Nachunternehmer vorgesehen gewesen waren, erscheint es außerdem naheliegend, dass diese in der Lage waren, die erforderlichen Leistungen noch rechtzeitig vor Ausstellungsbeginn zu erbringen, auch wenn sie erst kurzfristig am 11. bzw.
12. August 2009 beauftragt wurden. Auch eine nach Auffassung der ASt unzureichende Dokumentation der Beauftragung der einzelnen Transportunternehmen in der Vergabeakte bestätigt die Vermutung der ASt, es hätte bereits vor dem 10. August 2009 zumindest vertragsvorbereitende Kontakte der Ag geben müssen, nicht. Wenn es - so die Ag - keinen entsprechenden Schriftverkehr mit (potentiellen) Transporteuren gibt, kann darüber auch nichts in der Vergabeakte dokumentiert sein.

bb) Da die Transportleistungen aus (...), (...), (...), der (...) und (...) am 10. August 2009 noch nicht anderweitig vergeben oder sonst beauftragt worden waren, konnte deren Vergabe im Offenen Verfahren am 10. August 2009 ordnungsgemäß aufgehoben werden. Die Rechtmäßigkeit der Aufhebung des Vergabeverfahrens ergibt sich aus $\S 26 \mathrm{Nr} .1$ lit. a) VOL/A, da - unstreitig - kein Angebot eingegangen ist, das den Ausschreibungsbedingungen entspricht, vielmehr waren alle Angebote aus formalen Gründen zwingend von der Wertung auszuschließen (weil sie nicht die geforderten Eignungsnachweise enthielten, § $25 \mathrm{Nr}$. 2 Abs. 1 VOL/A (vgl. bereits oben), bzw. weil sie aufgrund der Beifügung eigener Allgemeiner Geschäftsbedingungen die Verdingungsunterlagen änderten oder ergänzten, § 25 Nr. 1 Abs. 1 lit. d) VOL/A (vgl. hierzu OLG München, Beschluss vom 21. Februar 2008, Verg 1/08)).

cc) Darüber hinaus durfte die Ag nach der (rechtmäßigen, s.o.) Aufhebung der Ausschreibung am 10. August 2009 die Aufträge für den Antransport der Exponate aus (...), (...), (...), der (...) und (...) nach (...) auch so wie geschehen an einzelne Spediteure vergeben. Denn selbst wenn man zugunsten der ASt unterstellt, dass die Schwellenwerte überschritten sind, war ein Verhandlungsverfahren ohne vorherige Öffentliche Vergabebekanntmachung gemäß $\S 3$ a Nr. 2 lit. d) VOL/A zulässig. Die Voraussetzungen dieser Norm sind hier erfüllt:

Dringlicher zwingender Grund iSd § 3a Nr. 2 lit. d) VOL/A, der die Ag dazu zwang, die Transportleistungen schnellstmöglich zu vergeben, war der Beginn der Ausstellung am (...) 2009. Der Antransport der Exponate (an den sich ein angemessener Zeitraum für das Auspacken und die Installation der Ausstellungsobjekte anschließt) sollte bis zum (...) abgeschlossen sein. Zumindest konnte somit bei Aufhebung des Vergabeverfahrens am 10. August 2009 unstreitig kein neues Vergabeverfahren innerhalb der Fristen gemäß § 18a VOL/A mehr durchgeführt werden. Doch selbst wenn man zugunsten der ASt darauf abstellt, dass ein neues Vergabeverfahren bereits unmittelbar nach der Auswertung der Angebote am 20. oder 21. Juli 2009 hätte durchgeführt werden müssen, hätten die Fristen gemäß $\S 18$ a VOL/A von der Ag nicht mehr eingehalten werden können. Die ASt hat zu etwaigen Vergaberechtsverstößen der Ag bei der Berechnung der einschlägigen Fristen nicht substantiiert vorgetragen. Zu berücksichtigen ist, dass die Ag nicht nur die Entscheidung über die Aufhebung hätte treffen müssen, sondern darüber hinaus zu entscheiden gehabt hätte, wie neu ausgeschrieben werden kann, so dass dieses Mal mit zuschlagsfähigen Angeboten zu rechnen ist; eine solche Neuausschrei- 
bung wäre zu konzipieren, europaweit bekannt zu machen (was gemäß § 17a Nr. 1 Abs. 3 VOL/A auch bei elektronisch erstellter und übersandter Bekanntmachung bis zu fünf Tage dauern kann), die den Bietern gesetzte Angebotsfrist hätte so bemessen werden müssen, dass die Interessenten ordnungsgemäße Angebote einreichen können (vgl. § 18a Nr. 1 Abs. 2 lit. b) VOL/A), die neu eingereichten Angebote hätten geöffnet, gewertet, die Informationsschreiben nach § 101a GWB hätten an die nicht berücksichtigten Bieter versandt werden müssen und schließlich hätte die sich hieran anschließende Wartefrist von mindestens 10 Kalendertagen beachtet werden müssen. Vor diesem Hintergrund wären die gemäß § 18a VOL/A zwingenden (Mindest-)Angebotsfristen auch ab dem 20. oder 21. Juli 2009 nicht mehr einzuhalten gewesen: So beträgt die gemäß $\S 18 a$ VOL/A kürzestmögliche Angebotsfrist selbst in Fällen besonderer Dringlichkeit gemäß § 18a Nr. 2 Abs. 2 S. 2 VOL/A mindestens 10 Tage, diese Frist ist jedoch nicht schon ab der Bekanntmachung, sondern vom Tag der Absendung der Aufforderung zur Angebotsabgabe an zu rechnen. Hinzu kommt also grundsätzlich noch ein voranzuschaltender Teilnahmewettbewerb, der seinerseits auch in Fällen besonderer Dringlichkeit die Einhaltung einer Frist für die Einreichung der Teilnahmeanträge von mindestens 10 Tagen ab dem Tag der Absendung der Bekanntmachung erfordert ( $\$ 18$ a Nr. 2 Abs. 1 VOL/A). Ein solcher Teilnahmewettbewerb wäre hier auch nicht mit dem Argument entbehrlich gewesen, dass (auf das aufgehobene Vergabeverfahren hin) bereits Angebote abgegeben worden waren und die Eignung der Bieter bereits von der Ag geprüft worden ist. Keiner der Bieter hatte nämlich die zum Nachweis der Eignung verlangten Nachweise vollständig vorgelegt, so dass deren Eignung bisher nicht positiv festgestellt werden konnte. Abgesehen davon ist davon auszugehen, und das verlangt auch gerade die ASt hinsichtlich des DIN ISO-Zertifikats für Kunsttransporte, dass die Ag im Falle einer Neuausschreibung mit Bekanntmachung ihre bisherigen Eignungsanforderungen abgeändert hätte - das Ergebnis der früheren Eignungsprüfung wäre daher ohnehin nicht ohne Weiteres auf ein neues förmliches Vergabeverfahren der Ag übertragbar gewesen. Wie die o.g. Berechnungen zeigen, hätten die Fristen gemäß § 18a VOL/A ab dem 21. Juli 2009 ebenfalls dann nicht gewahrt werden können, wenn man nicht auf den ursprünglich vorgesehenen Leistungsbeginn am 8. August, sondern auf ein späteres Datum bis zum Abschluss der zu erbringenden umfangreichen Leistungen spätestens am (...) 2009 abstellen würde, um nach Auspacken und Installation der auszustellenden Exponate den Ausstellungsbeginn am (...) 2009 einhalten zu können.

Auch die weiteren Voraussetzungen des $\S 3$ a Nr. 2 lit. d) VOL/A sind hier erfüllt. Die Ag konnte weder voraussehen, dass sie die Fristen nach $\S 18$ a VOL/A nicht einhalten kann, noch hatte sie die die Dringlichkeit begründenden Umstände zu vertreten. Denn das Scheitern der Vergabe der ausgeschriebenen Leistungen im Offenen Verfahren ist ausschließlich der Sphäre der Bieter zuzuordnen, die hier allesamt auszuschließende Angebote abgegeben haben. dd) Da die Ag ein Verhandlungsverfahren ohne vorherige Öffentliche Bekanntmachung durchführen durfte, musste sie auch nicht - wie die ASt es will - auf bestimmte Unternehmen zugehen und diese um Angebote bitten, sondern konnte sich auf diejenigen beschränken, bei denen ihr eine rechtzeitige Leistungserbringung möglich erschien. Die Ag ist in einem solchen Verfahren auch nicht verpflichtet, mit mindestens drei Unternehmen zu verhandeln. § 3a Nr. 1 Abs. 2 S. 3 VOL/A sieht dies ausdrücklich nur für Verhandlungsverfahren mit Öffentlicher Bekanntmachung vor, nicht jedoch, wenn eine solche Bekanntmachung wie hier gemäß $\S 3 a$ Nr. 2 lit. d) VOL/A unterbleiben darf. Gerade in den dringlichen Fällen - wie hier - wäre eine andere Vorgehensweise auch nicht aus allgemeinen wettbewerblichen Gründen angezeigt, da das Einholen von Angeboten von mehreren Unternehmen mit einem in solchen Eilfällen unzumutbar langen zeitlichen Aufwand verbunden wäre und die Verfahrenserleichterungen, die in solchen dringenden Fällen ausnahmsweise gerade zugelassen sind, im Ergebnis konterkarieren würde.

ee) Schließlich ist die ASt auch nicht durch etwaige Dokumentationsfehler der Ag in ihren Rechten verletzt.

Ein Dokumentationsmangel liegt hier bereits deshalb nicht vor, weil die hier verfahrensgegenständlichen Handlungen der Ag (Aufhebung und Neuvergabe, weil kein wertungsfähiges Angebot eingegangen ist) ohnehin noch nicht in der Vergabeakte niedergelegt sein konnten, sondern zwangsläufig erst im Rahmen des Nachprüfungsverfahrens dokumentiert werden konnten. Insoweit ist die Vorgehensweise der Ag durch entsprechende Anlagen zu ihren Schriftsätzen bereits hinreichend dokumentiert.

Aber selbst wenn die von der Ag durchgeführten Vergabeverfahren anhand der Vergabeakte nicht hinreichend iSd § 30 VOL/A nachvollzogen werden könnten, kann die ASt ihren Nachprüfungsantrag nur dann auf eine fehlende oder unzureichende Dokumentation stützen, wenn sich die diesbezüglichen Mängel gerade auch auf ihre Rechtsstellung im Vergabeverfahren nachteilig ausgewirkt haben (vgl. nur OLG Düsseldorf, Beschluss vom 22. August 2007, VII-Verg 27/07). Dies ist hier schon deshalb nicht der Fall, weil es hier nicht um die Nachholung unterlassener oder fehlerhafter Ermessenserwägungen durch die Ag geht, sondern um die objektiv zu beurteilende Rechtsfrage, ob die Voraussetzungen für ein Verhandlungsverfahren iSd § $3 a$ Nr. 2 VOL/A erfüllt waren oder nicht.

\section{III.}

Die Kostenentscheidung beruht auf $\S 128$ Abs. 3, Abs. 4 GWB und folgt dem Maß des Obsiegens und Unterliegens der Verfahrensbeteiligten. Bei der Kostenverteilung hat die Vergabekammer berücksichtigt, dass die ASt mit ihrem Begehren - Feststellung der Rechtswidrigkeit der Vergabe der Transportleistungen von sämtlichen Leihgebern - nicht in vollem Umfang durchgedrungen ist, sondern nur soweit es um die Leihgaben aus (...) 
(außer dem Exponat mit der Reg.-Nr. x0851 des Leihgebers 40) und (...) geht. Angesichts des Werts dieser Leistungen im Verhältnis zu dem der Ausstellungsobjekte im Übrigen erscheint die Auferlegung eines Kostenanteils von einem Fünftel zu Lasten der Ag als angemessen.

Eine höhere Kostenauferlegung zu Lasten der Ag aus Billigkeitserwägungen gemäß § 128 Abs. 3 S. 3, 5 GWB erscheint nicht angezeigt. Inwieweit die Ag selbst durch Verstöße gegen das Vergaberecht das Nachprüfungsverfahren veranlasst hat, wird bereits durch die o.g. Kostenquotelung entsprechend dem Maß ihres Unterliegens abgedeckt. Der Kostengrundsatz "der Unterliegende trägt insoweit die Kosten“ gilt auch im aktuellen Vergaberecht fort. Hierüber hinausgehende Billigkeitserwägungen, Verfahrenskosten, die aufgrund des Verschuldens eines Verfahrensbeteiligten entstanden sind, gerade diesem aufzuerlegen, kommt daher nur unter engen Voraussetzungen in Betracht, für deren Vorliegen hier keine Anhaltspunkte bestehen.

Die Zuziehung eines anwaltlichen Bevollmächtigten durch die Ag und die ASt war jeweils notwendig. Durch den Nachprüfungsantrag wurden nicht nur einfach gelagerte, auftragsbezogene Sach- und Rechtsfragen aufgeworfen, sondern hierüber hinausgehende nicht einfach gelagerte Rechtsfragen zur Durchführung des Vergabeverfahrens (vgl. BGH, Beschluss vom 26. September 2006, aaO).

Die Bg ist nicht an den Kosten des Verfahrens oder der Kostenerstattung zugunsten der ASt zu beteiligen, da sie nicht als "unterliegende" Partei anzusehen ist. Es ging der ASt nämlich allein darum, ob die Vorgehensweise der Ag, Transportleistungen an bestimmte Spediteure ohne Beteiligung der ASt zu vergeben, rechtmäßig war. Die ASt hat sich mit ihrem Nachprüfungsantrag somit nicht in einen Interessengegensatz zu der Bg gesetzt (vgl. OLG Düsseldorf, Beschlüsse vom 13. Juli 2005, VII-Verg 19/05, und vom 22. Oktober 2008, VII-Verg 48/08). Aus den gleichen Gründen entspricht es ebenfalls nicht der Billigkeit iSd § 128 Abs. 4 S. 2 GWB, der ASt die notwendigen Aufwendungen der Bg aufzuerlegen, soweit die ASt unterliegt (vgl. nur OLG Düsseldorf, Beschluss vom 22. Juli 2005, VII-Verg 28/05).

\section{IV.}

Gegen die Entscheidung der Vergabekammer ist die sofortige Beschwerde zulässig. Sie ist schriftlich innerhalb einer Frist von zwei Wochen, die mit der Zustellung der Entscheidung beginnt, beim Oberlandesgericht Düsseldorf - Vergabesenat -, Cecilienallee 3, 40474 Düsseldorf, einzulegen.

Die sofortige Beschwerde ist zugleich mit ihrer Einlegung zu begründen. Die Beschwerdebegründung muss die Erklärung enthalten, inwieweit die Entscheidung der Vergabekammer angefochten und eine abweichende Entscheidung beantragt wird, und die Tatsachen und Beweismittel angeben, auf die sich die Beschwerde stützt.
Die Beschwerdeschrift muss durch einen Rechtsanwalt unterschrieben sein. Dies gilt nicht für Beschwerden von juristischen Personen des öffentlichen Rechts.

Die sofortige Beschwerde hat aufschiebende Wirkung gegenüber der Entscheidung der Vergabekammer. Die aufschiebende Wirkung entfällt zwei Wochen nach Ablauf der Beschwerdefrist. Hat die Vergabekammer den Antrag auf Nachprüfung abgelehnt, so kann das Beschwerdegericht auf Antrag des Beschwerdeführers die aufschiebende Wirkung bis zur Entscheidung über die Beschwerde verlängern. (...) (Entscheidung von der Redaktion bearbeitet.) 